\title{
Greedy approximations for minimum submodular cover with submodular cost
}

\author{
Peng-Jun Wan · Ding-Zhu Du • Panos Pardalos • \\ Weili Wu
}

Received: 8 December 2007 / Published online: 19 June 2009

(C) Springer Science+Business Media, LLC 2009

\begin{abstract}
It is well-known that a greedy approximation with an integer-valued polymatroid potential function $f$ is $H(\gamma)$-approximation of the minimum submodular cover problem with linear cost where $\gamma$ is the maximum value of $f$ over all singletons and $H(\gamma)$ is the $\gamma$-th harmonic number. In this paper, we establish similar results for the minimum submodular cover problem with a submodular cost (possibly nonlinear) and/or fractional submodular potential function $f$.
\end{abstract}

Keywords Greedy approximations · Minimum submodular cover

The work of P.-J. Wan was partially supported by National Science Foundation of USA under grant CNS-0831831.

The work of D.-Z. Du was partially supported by a grant from the Research Grants Council of the Hong Kong Special Administrative Region, China [Project No. CityU 1165/04E] and also partially supported by National Science Foundation of USA under grant CCF-0728851.

The work of W. Wu was supported in part by National Science Foundation of USA under grant CCF-9208913.

P.-J. Wan

Department of Computer Science, Illinois Institute of Technology, Chicago, IL 60616, USA

e-mail:wan@cs.iit.edu

D.-Z. Du · W. Wu

Department of Computer Science, University of Texas at Dallas, Richardson, TX 75083, USA

D.-Z. Du

e-mail: dzdu@utdallas.edu

P. Pardalos $(\bowtie)$

Department of Industrial Engineering and System Science, University of Florida, Gainsville, FL 32611, USA

e-mail: pardalos@ufl.edu 


\section{Introduction}

Consider a ground set $E$ and a real function $f$ defined on $2^{E} . f$ is increasing if for $X \subset Y, f(X) \leq f(Y) . f$ is submodular if for any two subsets $X$ and $Y$ of $E$,

$$
f(X)+f(Y) \geq f(X \cup Y)+f(X \cap Y) .
$$

The marginal value of $Y \subseteq E$ with respect to $X \subseteq E$ is defined by

$$
\Delta_{Y} f(X)=f(X \cup Y)-f(X) .
$$

Similarly, the marginal value of an element $e \in E$ with respect to $X \subseteq E$ is defined by

$$
\Delta_{e} f(X)=f(X \cup\{e\})-f(X) .
$$

Both monotonicity and submodularity of a function $f$ can be characterized in terms of the marginal values (see, e.g., [1, 4-6]). $f$ is increasing if and only if $\Delta_{e} f(X) \geq 0$ for any $X \subseteq E$ and $e \in E \backslash X . f$ is submodular if and only if for any $X \subseteq E$ and different $a, b \in E \backslash X$.

$$
\Delta_{a} f(X) \geq \Delta_{a} f(X \cup\{b\}) .
$$

In addition, the following are equivalent:

- $f$ is increasing and submodular.

- For any $X, Y \subseteq E$,

$$
f(Y)-f(X) \leq \sum_{y \in Y \backslash X} \Delta_{y} f(X) .
$$

- For any $X \subseteq E$ and $a, b \in E \backslash X$,

$$
\Delta_{a} f(X) \geq \Delta_{a} f(X \cup\{b\}) .
$$

A submodular and increasing function $f$ is called a polymatroid function if $f(\emptyset)=0$. Suppose that $f$ is a polymatroid functions on $2^{E}$. Then, a set $X \subseteq E$ is said to be a submodular cover of $(E, f)$ if $f(X)=f(E)$. Suppose that both $f$ and $c$ are polymatroid functions on $2^{E}$. The minimization problem

$$
\min \{c(X): f(X)=f(E), X \subseteq E\}
$$

is known as a Minimum Submodular Cover with Submodular Cost (MSC/SC). A greedy approximation for it is described in Table 1 . We remark that $|E|$ may be not polynomial. In this case, we assume that there is polynomial-time oracle to compute an $x \in E$ with maximum $\Delta_{x} f(X) / c(x)$ for any $X \subset E$ with polynomial $|X|$. When $c$ is linear and $f$ is integer-valued, it is well-known that the algorithm GSC produces an $H(\gamma)$-approximation solution, where

$$
\gamma=\max _{x \in E} f(e)
$$


Table 1 Greedy algorithm for Minimum Submodular Cover

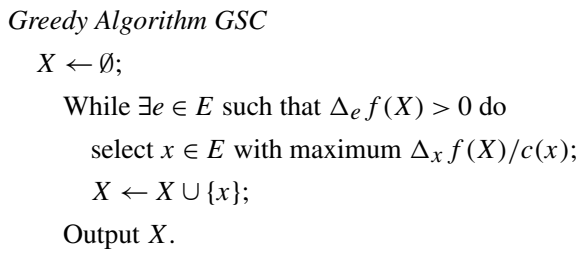

and

$$
H(k)=1+\frac{1}{2}+\cdots+\frac{1}{k}
$$

is the $k$-th Harmonic number [5]. In this paper, we establish similar results for the minimum submodular cover problem with a submodular cost (possibly nonlinear) and/or fractional submodular potential function $f$. Define the curvature of the submodular cost $c$ to be

$$
\rho=\min _{S: \text { min-cost cover }} \frac{\sum_{e \in S} c(e)}{c(S)} .
$$

Note that if $c$ is linear (i.e., modular), then $\rho=1$. This paper contains the following three contributions:

1. Analysis of the greedy algorithm for integral submodular cover with submodular cost. The charging argument is new and considerably simpler than all the known proofs for the linear-cost variant in the literature.

2. Analysis of the greedy algorithm for fractional submodular cover with submodular cost.

3. Application of the first result to obtaining a tighter approximation bound for a power assignment problem.

\section{Integral submodular cover}

In this section, we first show a general result on integral submodular cover, and then present a real-world problem as an example of submodular cover problem with submodular cost.

Theorem 2.1 If $f$ is integer-valued, then the greedy solution of GSC is a $\rho H(\gamma)$ approximation where $\gamma=\max _{e \in E} f(e)$.

Proof Let $x_{1}, x_{2}, \ldots, x_{k}$ be the sequence of elements selected by the greedy algorithm, and $S$ be a cover of minimum cost satisfying

$$
\sum_{e \in S} c(e)=\rho \cdot c(S) .
$$


We prove

$$
c(X) \leq \rho H(\gamma) \cdot c(S)
$$

by a charging argument. Set $X_{0}=\emptyset$, and $X_{i}=\left\{x_{j}: 1 \leq j \leq i\right\}$ for each $1 \leq i \leq k$. Denote $\mu_{0}=0$ and $\mu_{i}=\frac{c\left(x_{i}\right)}{\Delta_{x_{i}} f\left(X_{i-1}\right)}$ for each $1 \leq i \leq k$. The parameter $\mu_{i}$ is referred to as the average price per increment of coverage by $x_{i}$ for each $1 \leq i \leq k$. We claim that

$$
\mu_{0} \leq \mu_{1} \leq \mu_{2} \leq \cdots \leq \mu_{k} .
$$

Indeed, the first inequality is trivial. For any $1 \leq i<k$,

$$
\mu_{i}=\frac{c\left(x_{i}\right)}{\Delta_{x_{i}} f\left(X_{i-1}\right)} \leq \frac{c\left(x_{i+1}\right)}{\Delta_{x_{i+1}} f\left(X_{i-1}\right)} \leq \frac{c\left(x_{i+1}\right)}{\Delta_{x_{i+1}} f\left(X_{i}\right)}=\mu_{i+1},
$$

where the first inequality follows from the greedy rule and the second inequality follows from the submodularity of $f$. Thus, our claim holds. Now for iteration $i$ with $1 \leq i \leq k$, we charge each $e \in S$ with $\mu_{i}\left(\Delta_{e} f\left(X_{i-1}\right)-\Delta_{e} f\left(X_{i}\right)\right)$. Then, the total charge on each $e \in S$ is

$$
\sum_{i=1}^{k} \mu_{i}\left(\Delta_{e} f\left(X_{i-1}\right)-\Delta_{e} f\left(X_{i}\right)\right),
$$

and the total charge on $S$ is

$$
\sum_{e \in S} \sum_{i=1}^{k} \mu_{i}\left(\Delta_{e} f\left(X_{i-1}\right)-\Delta_{e} f\left(X_{i}\right)\right) .
$$

We claim that

1. $\sum_{i=1}^{k} c\left(x_{i}\right)$ is no more than the total charge on $S$.

2. The total charge on $e \in S$ is at most $H(\gamma) c(e)$.

The first claim is true because

$$
\begin{aligned}
& \sum_{i=1}^{k} c\left(x_{i}\right) \\
& \quad=\sum_{i=1}^{k} \mu_{i} \Delta_{x_{i}} f\left(X_{i-1}\right) \\
& \quad=\sum_{i=1}^{k} \mu_{i}\left(f\left(X_{i}\right)-f\left(X_{i-1}\right)\right) \\
& \quad=\sum_{i=1}^{k} \mu_{i}\left(\left(f(S)-f\left(X_{i-1}\right)\right)-\left(f(S)-f\left(X_{i}\right)\right)\right)
\end{aligned}
$$




$$
\begin{aligned}
& =\sum_{i=1}^{k} \mu_{i}\left(f(S)-f\left(X_{i-1}\right)\right)-\sum_{i=1}^{k} \mu_{i}\left(f(S)-f\left(X_{i}\right)\right) \\
& =\sum_{i=1}^{k} \mu_{i}\left(f(S)-f\left(X_{i-1}\right)\right)-\sum_{i=1}^{k-1} \mu_{i}\left(f(S)-f\left(X_{i}\right)\right) \quad\left(\text { as } f\left(X_{k}\right)=f(S)\right) \\
& =\sum_{i=1}^{k} \mu_{i}\left(f(S)-f\left(X_{i-1}\right)\right)-\sum_{i=2}^{k} \mu_{i-1}\left(f(S)-f\left(X_{i-1}\right)\right) \\
& =\sum_{i=1}^{k} \mu_{i}\left(f(S)-f\left(X_{i-1}\right)\right)-\sum_{i=1}^{k} \mu_{i-1}\left(f(S)-f\left(X_{i-1}\right)\right) \quad\left(\text { as } \mu_{0}=0\right) \\
& =\sum_{i=1}^{k}\left(\mu_{i}-\mu_{i-1}\right)\left(f(S)-f\left(X_{i-1}\right)\right) \\
& \leq \sum_{i=1}^{k}\left(\mu_{i}-\mu_{i-1}\right) \sum_{e \in S} \Delta_{e} f\left(X_{i-1}\right) \\
& =\sum_{e \in S} \sum_{i=1}^{k}\left(\mu_{i}-\mu_{i-1}\right) \Delta_{e} f\left(X_{i-1}\right) \\
& =\sum_{e \in S}\left(\sum_{i=1}^{k} \mu_{i} \Delta_{e} f\left(X_{i-1}\right)-\sum_{i=1}^{k} \mu_{i-1} \Delta_{e} f\left(X_{i-1}\right)\right) \\
& =\sum_{e \in S}\left(\sum_{i=1}^{k} \mu_{i} \Delta_{e} f\left(X_{i-1}\right)-\sum_{i=2}^{k} \mu_{i-1} \Delta_{e} f\left(X_{i-1}\right)\right) \quad\left(\text { as } \mu_{0}=0\right) \\
& =\sum_{e \in S}\left(\sum_{i=1}^{k} \mu_{i} \Delta_{e} f\left(X_{i-1}\right)-\sum_{i=1}^{k-1} \mu_{i} \Delta_{e} f\left(X_{i}\right)\right) \\
& =\sum_{e \in S}\left(\sum_{i=1}^{k} \mu_{i} \Delta_{e} f\left(X_{i-1}\right)-\sum_{i=1}^{k} \mu_{i} \Delta_{e} f\left(X_{i}\right)\right) \quad\left(\text { as } \Delta_{e} f\left(X_{k}\right)=0\right) \\
& =\sum_{e \in S} \sum_{i=1}^{k} \mu_{i}\left(\Delta_{e} f\left(X_{i-1}\right)-\Delta_{e} f\left(X_{i}\right)\right) \text {. }
\end{aligned}
$$

Next, we prove the second claim. Consider an arbitrary element $e \in S$. Let $l$ be the first $i$ such that $\Delta_{e} f\left(X_{i}\right)=0$. For each $1 \leq i \leq l$, by the greedy rule,

$$
\mu_{i}=\frac{c\left(x_{i}\right)}{\Delta_{x_{i}} f\left(X_{i-1}\right)} \leq \frac{c(e)}{\Delta_{e} f\left(X_{i-1}\right)} .
$$


Hence,

$$
\begin{aligned}
& \sum_{i=1}^{k} \mu_{i}\left(\Delta_{e} f\left(X_{i-1}\right)-\Delta_{e} f\left(X_{i}\right)\right) \\
& =\sum_{i=1}^{l-1} \mu_{i}\left(\Delta_{e} f\left(X_{i-1}\right)-\Delta_{e} f\left(X_{i}\right)\right)+\mu_{l} \Delta_{e} f\left(X_{l-1}\right) \\
& \text { (as } \left.\Delta_{e} f\left(X_{i}\right)=0 \text { with } i \geq l\right) \\
& \leq \sum_{i=1}^{l-1} \frac{c(e)\left(\Delta_{e} f\left(X_{i-1}\right)-\Delta_{e} f\left(X_{i}\right)\right)}{\Delta_{e} f\left(X_{i-1}\right)}+\frac{c(e) \Delta_{e} f\left(X_{l-1}\right)}{\Delta_{e} f\left(X_{l-1}\right)} \\
& =c(e)\left(1+\sum_{i=1}^{l-1} \frac{\Delta_{e} f\left(X_{i-1}\right)-\Delta_{e} f\left(X_{i}\right)}{\Delta_{e} f\left(X_{i-1}\right)}\right) \\
& \leq c(e)\left(1+\sum_{i=1}^{l-1} \sum_{j=0}^{\Delta_{e} f\left(X_{i-1}\right)-\Delta_{e} f\left(X_{i}\right)-1} \frac{1}{\Delta_{e} f\left(X_{i-1}\right)-j}\right) \\
& =c(e)\left(1+\sum_{i=1}^{l-1} \sum_{j=\Delta_{e} f\left(X_{i}\right)+1}^{\Delta_{e} f\left(X_{i-1}\right)} \frac{1}{j}\right) \\
& =c(e)\left(1+\sum_{j=\Delta_{e} f\left(X_{l-1}\right)+1}^{\Delta_{e} f\left(X_{0}\right)} \frac{1}{j}\right) \\
& =c(e)\left(1+\sum_{j=\Delta_{e} f\left(X_{l-1}\right)+1}^{\Delta_{e} f(\emptyset)} \frac{1}{j}\right) \\
& =c(e)\left(1+\sum_{j=1}^{\Delta_{e} f(\emptyset)} \frac{1}{j}-\sum_{j=1}^{\Delta_{e} f\left(X_{l-1}\right)} \frac{1}{j}\right) \\
& =c(e)\left(1+H\left(\Delta_{e} f(\emptyset)\right)-H\left(\Delta_{e} f\left(X_{l-1}\right)\right)\right) \\
& \leq c(e)(1+H(\gamma)-H(1)) \\
& =c(e) H(\gamma) \text {. }
\end{aligned}
$$

So, the second claim also holds. The two claims imply that

$$
\sum_{i=1}^{k} c\left(x_{i}\right) \leq H(\gamma) \sum_{e \in S} c(e)=\rho H(\gamma) \cdot c(S) .
$$


By the submodularity of $c$, we have

$$
c(X) \leq \sum_{i=1}^{k} c\left(x_{i}\right) \leq \rho H(\gamma) \cdot c(S) .
$$

Thus, the theorem follows.

Next, we give an application of above theorem. Let $D=(V, A ; w)$ be any arcweighted digraph with $w(e)>0$ for any $e \in A$. Any subgraph $H$ induces a power assignment $p_{H}$ to $V$ defined as follows: For each node $u \in V$ which is the tail node of at least one arc in $H, p_{H}(u)=\max _{u v \in H} w(u v)$; otherwise, $p_{H}(u)=0$. The power cost of $H$ is defined by $p(H)=\sum_{v \in V} p_{H}(v)$. We will treat each subgraph $H$ of $D$ as a subset of $\operatorname{arcs}$ in $A$. For any $B \subseteq A$, the power cost of $B$, denoted by $p(B)$, is the power cost of the subgraph of $D$ induced by $B$. It's easy to verify that $p$ is an increasing and submodular function on $2^{A}$ and $p(\emptyset)=0$. The undirected version of a digraph $D$, denoted by $\bar{D}$, is the undirected graph obtained from $D$ by ignoring the orientations of the arcs in $D$ and then removing multiple edges between any pair of nodes. $D$ is said to be weakly-connected if $\bar{D}$ is connected. The bidirected version of an undirected graph $G$, denoted by $\vec{G}$, is the digraph obtained from $G$ by replacing every edge $u v$ of $G$ with two oppositely oriented arcs $u v$ and $v u$. A digraph $D=(V, A)$ is said to be bidirected if $u v \in A$ implies $v u \in A$. Now, we introduce the problem Min-Power Spanning Tree in Digraphs. An instance of this problem is an arc-weighted, connected and bidirected graph $D=(V, A ; w)$ with $w(e)>0$ for any $e \in A$. The objective is to find a spanning tree $T$ of $\bar{D}=(V, E)$ with minimum $p(\vec{T})$. This problem arises from the algorithmic study of maximum-life power scheduling for connectivity in wireless ad hoc networks [2]. It is at least as hard as SET COVER [3] and a $2(1+\ln (n-1))$-approximation for this problem was reported in [2]. In this section, we will apply Theorem 2.1 to obtain a greedy $2 H(\Delta)$-approximation, where $\Delta$ is the maximum degree of $\bar{D}$ (or equivalently, the maximum in-degree or out-degree of $D$ ). The problem Min-Power Spanning Tree in Digraphs can be cast as a problem of MSC. Indeed, let $r$ be the graphic matroid rank of $\bar{D}$, which is defined as follows. For any $F \subseteq E$, denote by $\kappa(V, F)$ the number of connected components of the graph $(V, F)$, then

$$
r(F)=|V|-\kappa(V, F) .
$$

For any $F \subseteq E$, define $c(F)$ to be the power cost of the bidirected version of the graph $(V, F)$. Then, both $r$ and $c$ are increasing and submodular functions on $2^{E}$ with $r(\emptyset)=c(\emptyset)=0$, and $(V, F)$ is a connected spanning graph of $\bar{D}$ if and only if $r(F)=|V|-1=r(E)$. Thus, the problem is exactly

$$
\min \{c(F): r(F)=r(E), F \subseteq E\} .
$$

However, if we apply the greedy algorithm naively with $E$ as the ground set, Theorem 2.1 can only imply an upper bound $\Delta$ on the approximation ratio. Indeed, when $E$ is the ground set, $\gamma=1$ as $r$ is a matroid rank, but the curvature of $c$ can be as large as $\Delta$. For example, we consider an instance of $D$ in which $V=\left\{v_{0}, v_{1}, \ldots, v_{n}\right\}$, 
$A=\left\{v_{0} v_{i}: 1 \leq i \leq n\right\} \cup\left\{v_{i} v_{0}: 1 \leq i \leq n\right\}, w\left(v_{0} v_{i}\right)=1$ and $w\left(v_{i} v_{0}\right)=\varepsilon$ for $1 \leq i \leq n$. Then, $\bar{D}$ is a star, which is also the (unique) optimal solution, and $\Delta=n$. Since $c(\bar{D})=p(D)=1+\Delta \varepsilon$ and $c(e)=1+\varepsilon$ for every edge $e$ of $\bar{D}$, the curvature of $c$ is $\frac{\Delta(1+\varepsilon)}{1+\Delta \varepsilon}$ which tends to $\Delta$ as $\varepsilon$ tends to 0 . Thus, the approximation ratio is bounded by $\Delta$. In the following, we describe how to apply the same Theorem 2.1 to obtain a greedy logarithmic approximation for the above problem. Instead of choosing $E$ as the ground set, we choose the set $\mathcal{S}$ of all stars in $\bar{D}$ as the ground set. The ground set $\mathcal{S}$ may have exponential cardinality, but the greedy algorithm only uses it implicitly. We can extend both $r$ and $c$ to $2^{\mathcal{S}}$ in the straightforward manner. Specifically, for any subset of stars $\mathcal{F} \subseteq \mathcal{S}, r(\mathcal{F})$ is defined to be the graphic matroid rank of the union of the stars in $\mathcal{F}$, and $c(\mathcal{F})$ is defined to be the power cost of the bidirected version of the union of the stars in $\mathcal{F}$. Then, both $r$ and $c$ are also increasing and submodular functions on $2^{\mathcal{S}}$ with $r(\emptyset)=c(\emptyset)=0$, and the union of the stars in a subset $\mathcal{F} \subseteq \mathcal{S}$ is a connected spanning graph of $\bar{D}$ if and only if $r(\mathcal{F})=|V|-1=r(\mathcal{S})$. Thus, the problem can be formulated as

$$
\min \{c(\mathcal{F}): r(\mathcal{F})=r(\mathcal{S}), \mathcal{F} \subseteq \mathcal{S}\}
$$

For such formulation, we claim that (1) $\gamma=\max _{S \in \mathcal{S}} r(S)=\Delta$ and (2) the curvature $\rho$ of $c$ is at most 2. The first claim follows from the fact that for any star $S$ with the degree of the center equal to $d, r(S)=d$. The second claim follows from a decomposition argument. Let $T$ be an optimal minimum spanning tree. Root $T$ at an arbitrary node, and let $U$ be the set of internal nodes in $T$ and the root of $T$. For each $u \in U$, let $T_{u}$ be the star consisting of the edges between $u$ and its children in $T$. Then, $\left\{T_{u}: u \in U\right\}$ is a partition of $T$ into stars. It's easy to show that

$$
\sum_{u \in U} c\left(T_{u}\right) \leq 2 c(T) .
$$

Thus, the second claim holds. By Theorem 2.1, the approximation ratio of the greedy algorithm is at most $2 H(\Delta)$. In the remaining of this section, we describe a polynomial-time oracle which computes a star $S \subset \bar{D}$ with maximum $\Delta_{S} r(H) / c(S)$ for any disconnected spanning subgraph $H$ of $\bar{D}$. Suppose that $H$ is a disconnected spanning subgraph of $\bar{D}$. Let $V^{\prime}$ be the set of nodes $v$ with at least one neighbor in a different connected component of $H$ from $v$. For each $v \in V^{\prime}$, let $\Gamma(v)$ be the set of neighbors of $v$ in $\bar{D}$ not belonging to the connected component of $H$ containing $v$, and let

$$
Q(v)=\{w(v u): u \in \Gamma(v)\}
$$

For each $q \in Q(v)$, let

$$
\Gamma(v, q)=\{u \in \Gamma(v): w(v u) \leq q\}
$$

In each connected component of $H$ which contains at least one node in $\Gamma(v, q)$, choose a node $u \in \Gamma(v, q)$ with minimum $w(u v)$. Let $u_{1}, u_{2}, \ldots, u_{l}$ be those chosen nodes with

$$
w\left(u_{1} v\right) \leq w\left(u_{2} v\right) \leq \cdots \leq w\left(u_{l} v\right) .
$$


Compute $1 \leq j \leq l$ maximizing $j /\left(q+\sum_{i=1}^{j} w\left(u_{i} v\right)\right)$, and let $S(v, q)$ be the star connecting $v$ to $u_{1}, u_{2}, \ldots, u_{j}$. Then, compute $q \in Q(v)$ maximizing $\Delta_{S(v, q)} r(H) /$ $c(S(v, q))$, and let $S(v)$ be the star $S(v, q)$. Finally, compute $v \in V^{\prime}$ maximizing $\Delta_{S(v)} r(H) / c(S(v))$, and let $S$ be the star $S(v)$. We claim that $S$ is a star in $\bar{D}$ with maximum $\Delta_{S} r(H) / c(S)$. Indeed, consider an optimal star $S^{\prime}$ with head $v_{0}$ and $j$ leaves $v_{1}, v_{2}, \ldots, v_{j}$ satisfying

$$
w\left(v_{1} v_{0}\right) \leq w\left(v_{2} v_{0}\right) \leq \cdots \leq w\left(v_{j} v_{0}\right)
$$

Clearly, all the nodes in $S^{\prime}$ must belong to distinct connected components of $H$, and hence all the leaves of $S^{\prime}$ must belong to $\Gamma\left(v_{0}\right)$. Let

$$
q^{\prime}=\max _{1 \leq i \leq j} w\left(v_{0} v_{i}\right)
$$

Then, $q^{\prime} \in Q\left(v_{0}\right)$ and all the leaves of $S^{\prime}$ belong to $\Gamma\left(v_{0}, q^{\prime}\right)$. Since

$$
\frac{\Delta_{S^{\prime}} r(H)}{c\left(S^{\prime}\right)}=\frac{j}{q^{\prime}+\sum_{i=1}^{j} w\left(v_{i} v_{0}\right)}
$$

the sum $\sum_{i=1}^{j} w\left(v_{i} v_{0}\right)$ must achieve the minimum over all sets of $j$ nodes in $\Gamma\left(v_{0}, q^{\prime}\right)$ belonging to distinct connected components of $H$. Let $u_{1}, u_{2}, \ldots, u_{j}$ be the first $j$ nodes chosen from $\Gamma\left(v_{0}, q^{\prime}\right)$ as in the above oracle. By the standard swapping argument, we can show that $w\left(v_{i} v_{0}\right)=w\left(u_{i} v_{0}\right)$ for each $1 \leq i \leq j$. Hence,

$$
\frac{\Delta_{S^{\prime}} r(H)}{c\left(S^{\prime}\right)} \leq \frac{\Delta_{S\left(v_{0}, q^{\prime}\right)} r(H)}{c\left(S\left(v_{0}, q^{\prime}\right)\right)} \leq \frac{\Delta_{S} r(H)}{c(S)} .
$$

So, our claim holds.

\section{Fractional submodular cover}

In this section, we present a general result on fractional submodular cover.

Theorem 3.1 Suppose that $f$ is fractional and $f(E) \geq$ opt where opt is the cost of a minimum submodular cover. If in each iteration of the Greedy Algorithm GSC, the selected $x$ always satisfies that $\Delta_{x} f(X) / c(x) \geq 1$, then the greedy solution is a $(1+\rho \ln (f(E) /$ opt $))$-approximation.

Proof Let $x_{1}, x_{2}, \ldots, x_{k}$ be the sequence of elements selected by the greedy algorithm, and $S$ be a cover of minimum cost satisfying

$$
\sum_{e \in S} c(e)=\rho \cdot c(S)=\rho \cdot o p t
$$

Set $X_{0}=\emptyset$, and $X_{i}=\left\{x_{j}: 1 \leq j \leq i\right\}$ for each $1 \leq i \leq k$. For each $0 \leq i \leq k$, let $\ell_{i}=f(S)-f\left(X_{i}\right)$ be the "uncoverage" at the end of iteration $i$. We first claim that 
for each $1 \leq i \leq k$,

$$
c\left(x_{i}\right) \leq \min \left\{1, \frac{\rho \cdot o p t}{\ell_{i-1}}\right\}\left(\ell_{i-1}-\ell_{i}\right) .
$$

Indeed,

$$
\begin{aligned}
& \frac{\ell_{i-1}-\ell_{i}}{c\left(x_{i}\right)} \\
& =\frac{\Delta_{x_{i}} f\left(X_{i-1}\right)}{c\left(x_{i}\right)} \\
& \geq \max _{e \in S} \frac{\Delta_{e} f\left(X_{i-1}\right)}{c(e)} \\
& \geq \frac{\sum_{e \in S} \Delta_{e} f\left(X_{i-1}\right)}{\sum_{e \in S} c(e)} \\
& \geq \frac{f(S)-f\left(X_{i-1}\right)}{\sum_{e \in S} c(e)} \\
& =\frac{f(S)-f\left(X_{i-1}\right)}{\rho \cdot o p t} \\
& =\frac{\ell_{i-1}}{\rho \cdot o p t} .
\end{aligned}
$$

Thus,

$$
c\left(x_{i}\right) \leq \frac{\rho \cdot o p t}{\ell_{i-1}}\left(\ell_{i-1}-\ell_{i}\right) .
$$

The other inequality $c\left(x_{i}\right) \leq \ell_{i-1}-\ell_{i}$ follows from the assumption that $\Delta_{x_{i}} f\left(X_{i-1}\right) /$ $c\left(x_{i}\right) \geq 1$. Since

$$
f(E)=\ell_{0}>\ell_{1}>\cdots>\ell_{k}=0
$$

and $f(E) \geq o p t$ by assumption, there exists a unique index $t$ satisfying $\ell_{t} \geq o p t>$ $\ell_{t+1}$. Using the inequalities

$$
c\left(x_{i}\right) \leq \frac{\rho \cdot o p t}{\ell_{i-1}}\left(\ell_{i-1}-\ell_{i}\right)
$$

for $1 \leq i \leq t+1$, we have

$$
\begin{aligned}
& \sum_{i=1}^{t} c\left(x_{i}\right)+\frac{\ell_{t}-o p t}{\ell_{t}-\ell_{t+1}} c\left(x_{t+1}\right) \\
& \quad \leq \rho \cdot o p t\left(\sum_{i=1}^{t} \frac{\ell_{i-1}-\ell_{i}}{\ell_{i-1}}+\frac{\ell_{t}-o p t}{\ell_{t}}\right)
\end{aligned}
$$




$$
\begin{aligned}
& \leq \rho \cdot o p t \int_{o p t}^{\ell_{0}} \frac{1}{y} d y \\
& =\rho \cdot o p t \ln \frac{\ell_{0}}{o p t} \\
& =\rho \cdot o p t \ln \frac{f(E)}{o p t} .
\end{aligned}
$$

Using the inequalities $c\left(x_{i}\right) \leq \ell_{i-1}-\ell_{i}$ for $t+1 \leq i \leq k$, we have

$$
\begin{aligned}
& \frac{o p t-\ell_{t+1}}{\ell_{t}-\ell_{t+1}} c\left(x_{t+1}\right)+\sum_{i=t+2}^{k} c\left(x_{i}\right) \\
& \leq o p t-\ell_{t+1}+\sum_{i=t+2}^{k}\left(\ell_{i-1}-\ell_{i}\right) \\
& =\text { opt }-\ell_{k} \\
& =\text { opt. }
\end{aligned}
$$

Hence,

$$
\begin{aligned}
& \sum_{i=1}^{k} c\left(x_{i}\right) \\
& \quad=\sum_{i=1}^{t} c\left(x_{i}\right)+\frac{\ell_{t}-o p t}{\ell_{t}-\ell_{t+1}} c\left(x_{t+1}\right)+\frac{o p t-\ell_{t+1}}{\ell_{t}-\ell_{t+1}} c\left(x_{t+1}\right)+\sum_{i=t+2}^{k} c\left(x_{i}\right) \\
& \quad \leq \rho \cdot o p t \ln \frac{f(E)}{o p t}+o p t \\
& \quad=\left(1+\rho \ln \frac{f(E)}{o p t}\right) o p t .
\end{aligned}
$$

Thus, the theorem follows.

In the study of Steiner trees, several greedy approximations have a fractional submodular potential function. In such a case, the above theorem may apply.

\section{References}

1. Baudis, G., Gröpl, C., Hougardy, S., Nierhoff, T., Prömel, H.J.: Approximating minimum spanning sets in hypergraphs and polymatroids. Technical Report, Humboldt-Universitt zu Berlin (2000)

2. Calinescu, G., Kapoor, S., Olshevsky, A., Zelikovsky, A.: Network lifetime and power assignment in ad hoc wireless networks. In: Lectures Notes in Computer Science, vol. 2832, pp. 114-126. Springer, Berlin (2003) 
3. Feige, U.: A threshold of $\ln n$ for approximating set cover. In: Proc. the Twenty-Eighth Annual ACM Symp. Theory of Computing, pp. 314-318 (1996)

4. Fujito, T.: Approximation algorithms for submodular set cover with application. IEICE Trans. Inf. Syst. E83-D(3), 480-487 (2000)

5. Nemhauser, G.L., Wolsey, L.A.: Integer and Combinatorial Optimization. Wiley, New York (1999)

6. Wolsey, L.A.: An analysis of the greedy algorithm for submodular set covering problem. Combinatorica 2(4), 385-393 (1982) 